\title{
Investigation of Local Structure and Enhanced Thermal Stability of Ir-doped PdRu Nanoparticles for the Three-way Catalytic Application
}

Akhil Tayal, ${ }^{\dagger}$ Okkyun Seo ${ }^{\ddagger}, \perp$ Jaemyung Kim,${ }^{\ddagger}$ Kohei Kusada, ${ }^{\text {II }}$ Hiroshi Kitagawa, ${ }^{\text {II }}$ and Osami Sakata $\$, \S, \|$

$\dagger$ Deutsches Elektronen-Synchrotron, Notkestrasse 85, 22607 Hamburg Germany. ¥Synchrotron X-ray Group, Research Center for Advanced Measurement and Characterization, National Institute for Materials Science, 1-1-1 Kouto, Sayo, Hyogo 6795148, Japan.

IDivision of Chemistry, Graduate School of Science, Kyoto University, Kitashirakawa Oiwake-cho, Sakyo-ku, Kyoto 606-8502, Japan.

$\S$ Department of Materials Science and Engineering, Tokyo Institute of Technology, Nagatsuta, Midori, Yokohama 226-8502, Japan.

॥Center for Synchrotron Radiation Research, Japan Synchrotron Radiation Research Institute (JASRI),1-1-1 Kouto, Sayo, Hyogo 679-5198, Japan. 


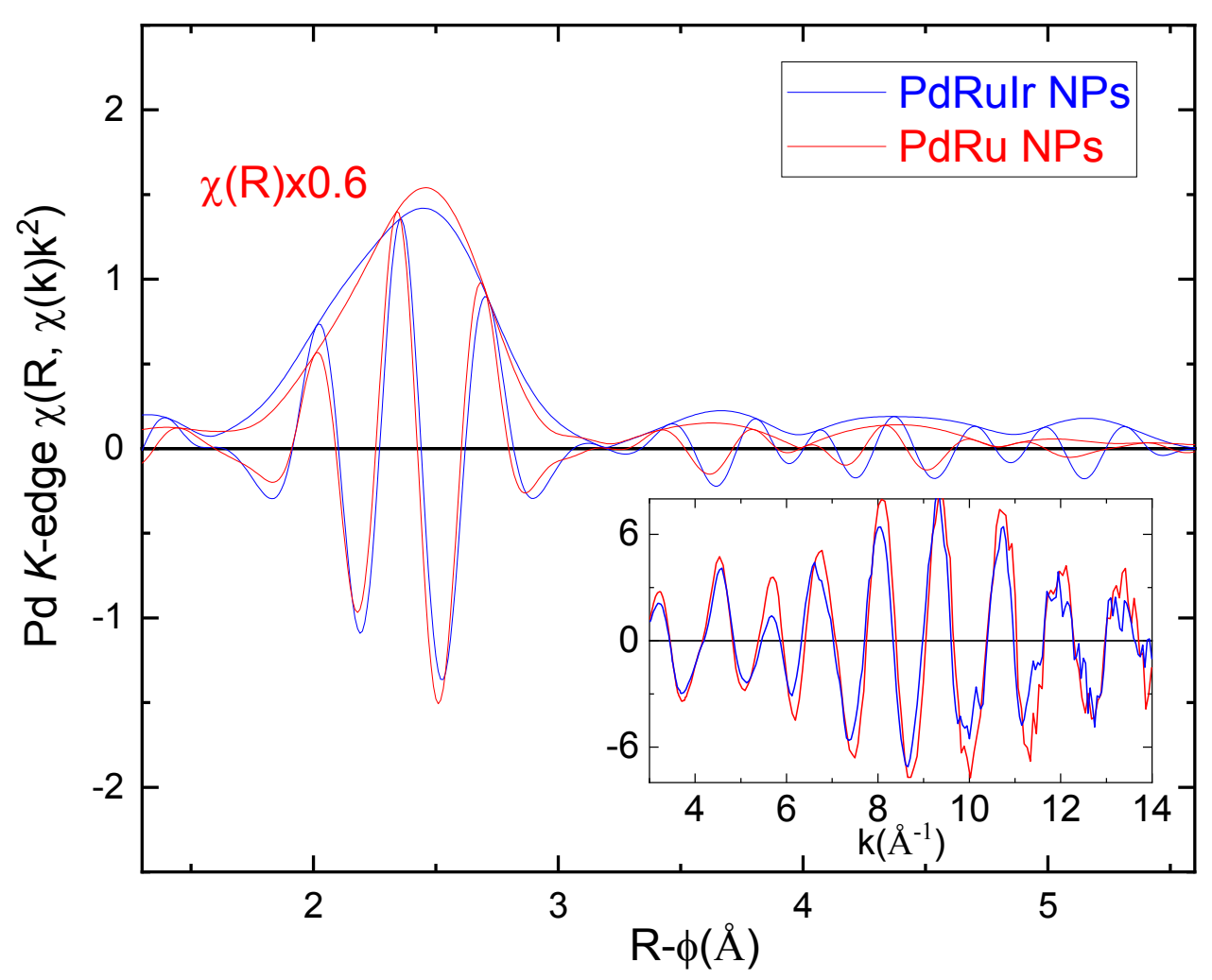

Figure S1. Comparison of Pd K-edge EXAFS $\chi(\mathrm{k}) \mathrm{k}^{2}$ (inset) and corresponding Fourier transform $(\chi(\mathrm{R}))$ of PdRu NPs and PdRuIr NPs. The FT spectra of PdRu NPs was scaled down to compare the asymmetry, peak width and variation in the node position with PdRuIr NPs. A small variation in the node position in the $\chi(\mathrm{k})$ spectra is also evident. 


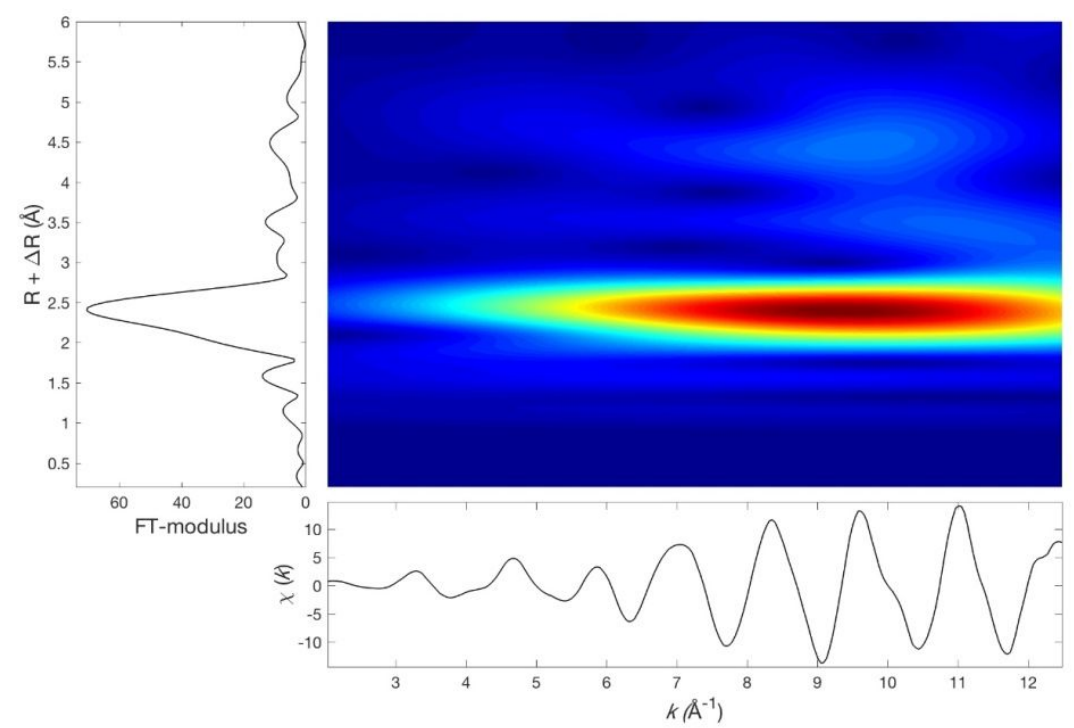

(a)

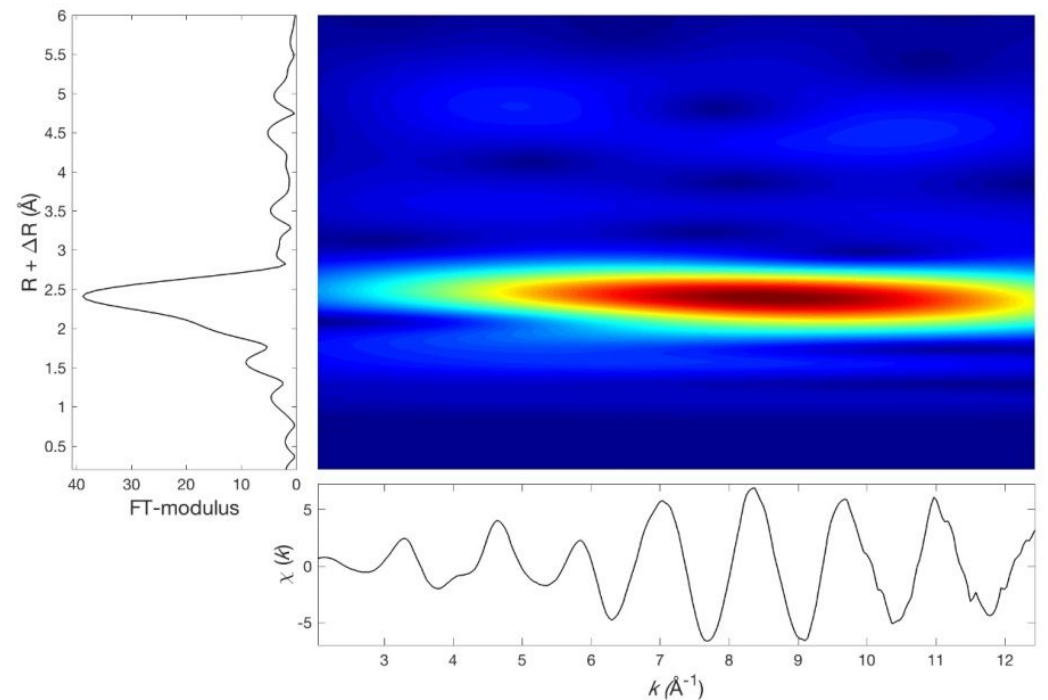

(b)

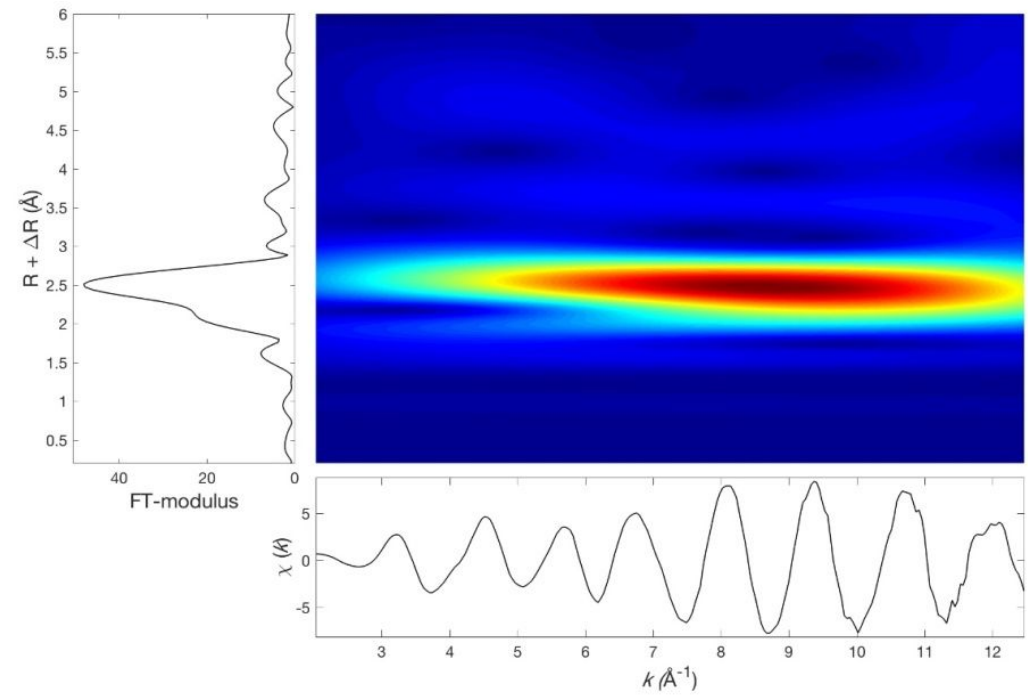

(c)

Figure S2. CCWT, $\chi(\mathrm{k})$, and Fourier transform spectra measured at Ru K-edge $(\mathrm{a}, \mathrm{b})$ and Pd K-edge (b) for Ru NPs (a) and PdRu NPs (b,c). 


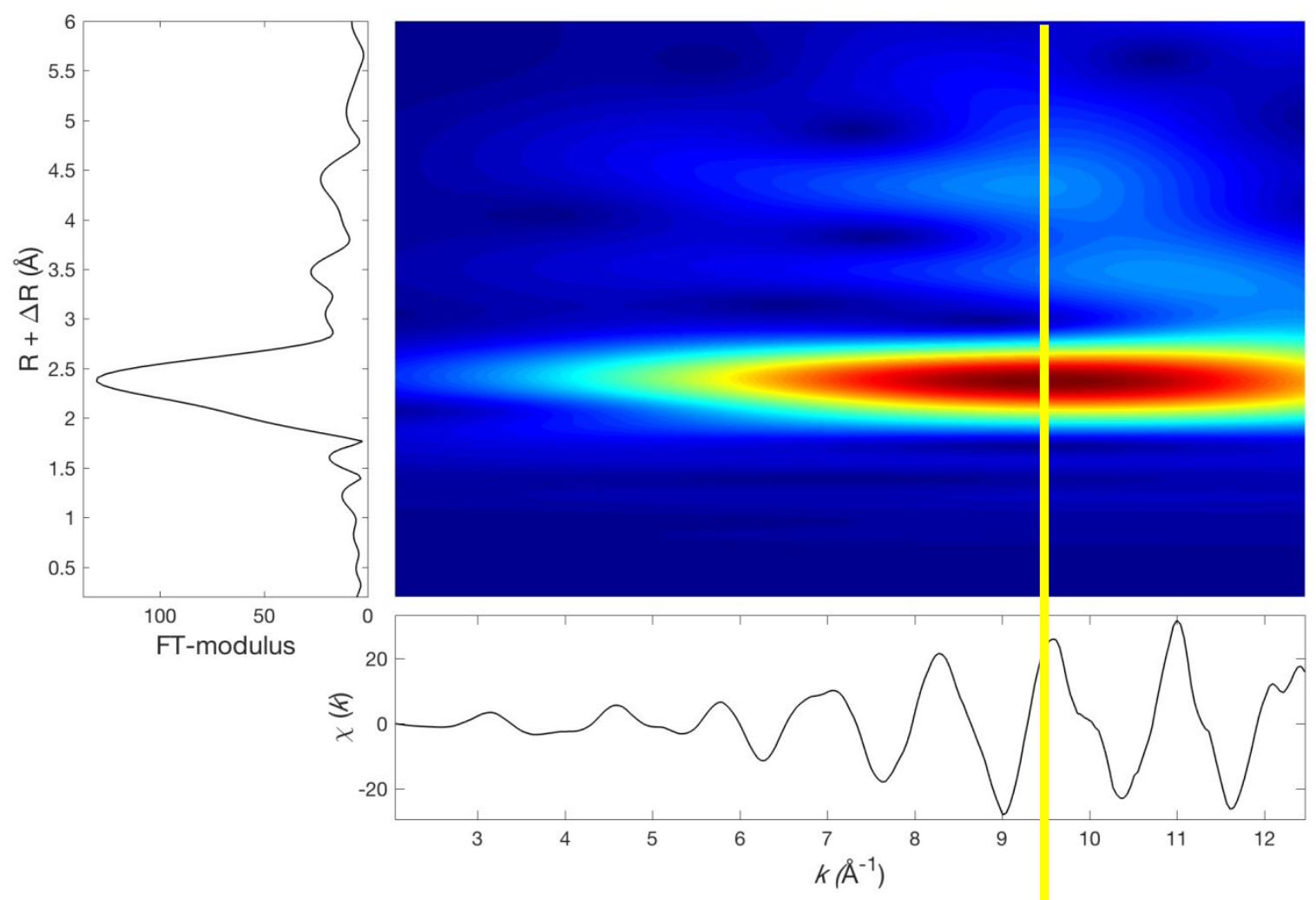

(a)

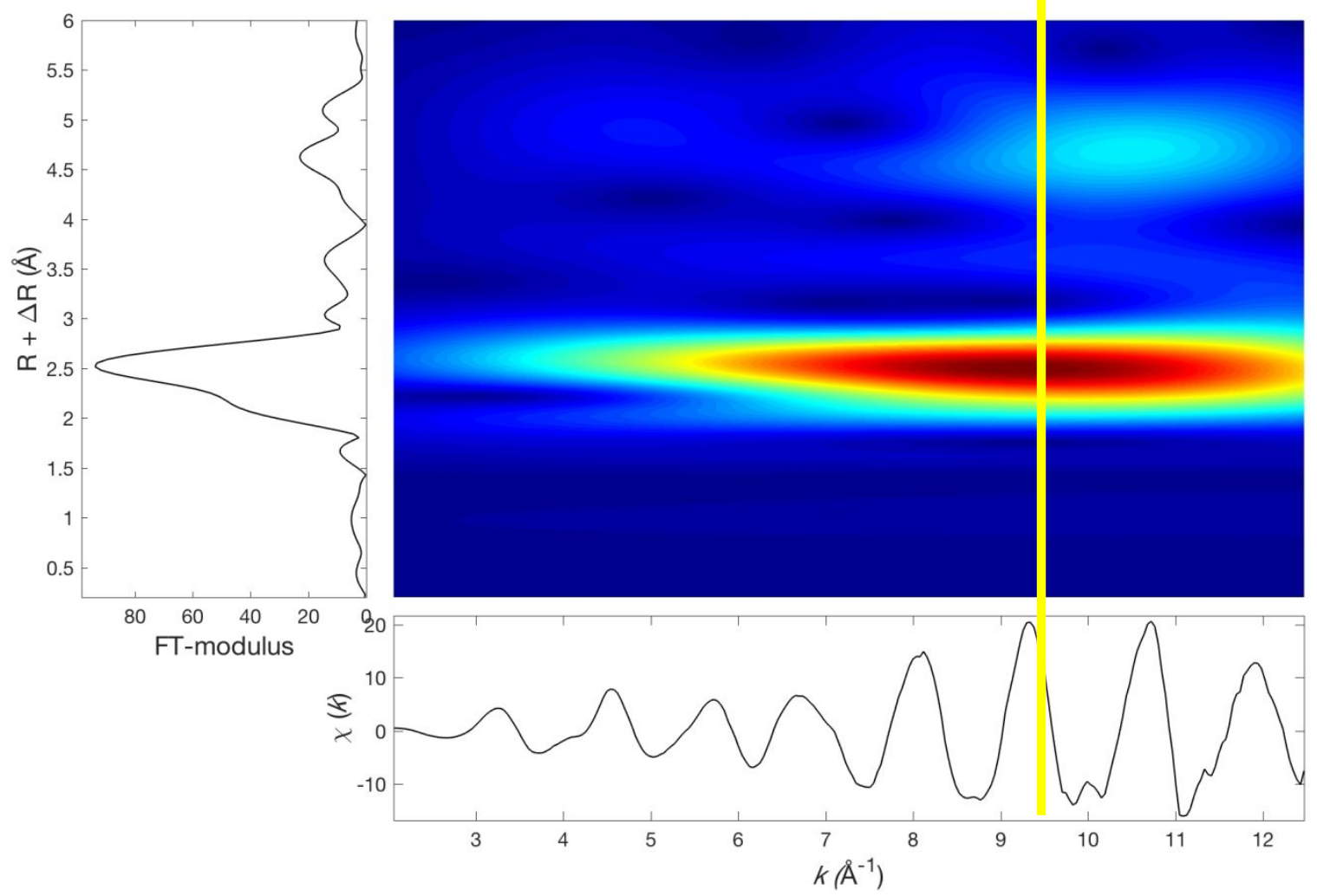

(b)

Figure S3 CCWT, $\chi(\mathrm{k})$, and Fourier transform spectra measured at Ru K-edge (a) and Pd Kedge (b) for $\mathrm{Ru}(\mathrm{a})$ and $\mathrm{Pd}(\mathrm{b})$ references. The yellow line indicates the peak of the wavelet ridge. 


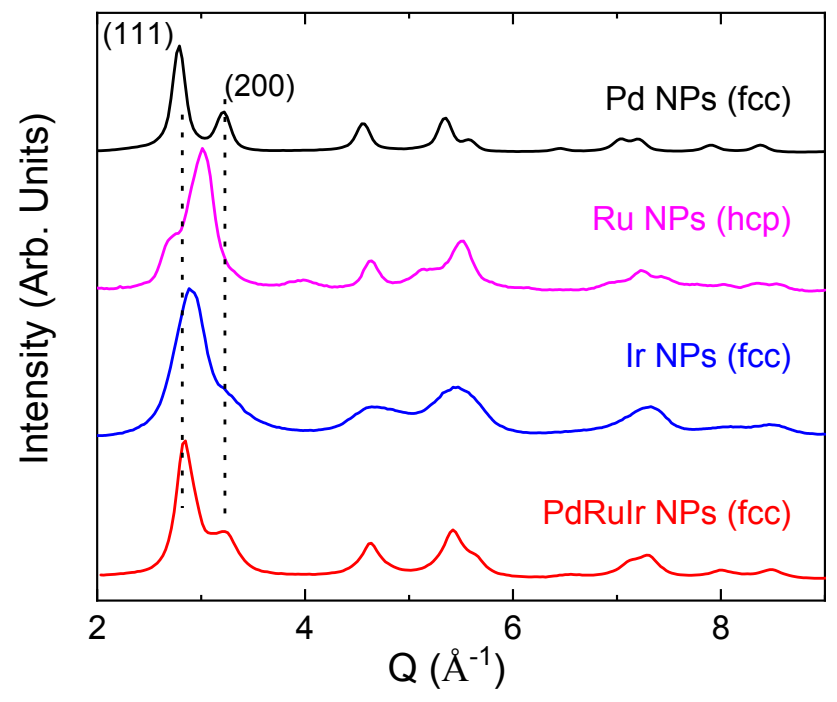

Figure S4 Synchrotron X-ray diffraction of fcc Pd, fcc Ir and hep Ru NPs are compared with PdRuIr NPs. It can be seen that relative intensity of 111 reflection is higher than 200 reflection for the fcc structure. It can be seen that for the PdRuIr NPs the intensity of 111 and 200 reflections follow the similar trend as observed for the fcc NPs.

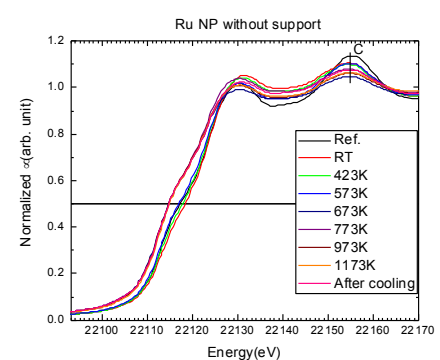

(a)

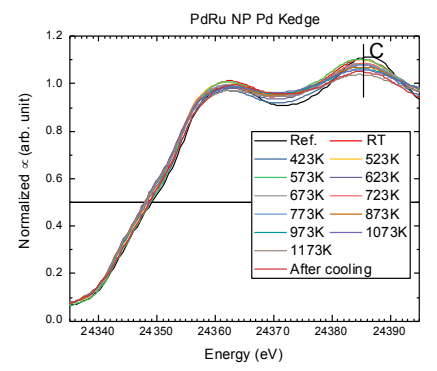

(d)

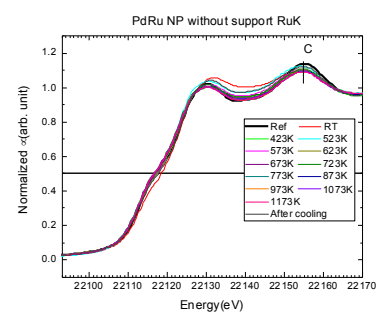

(b)

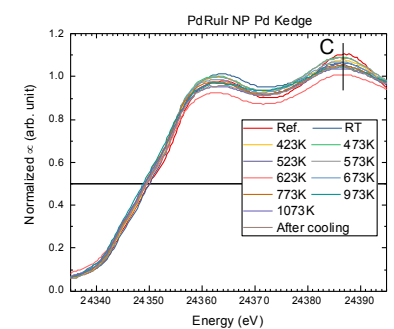

(e)

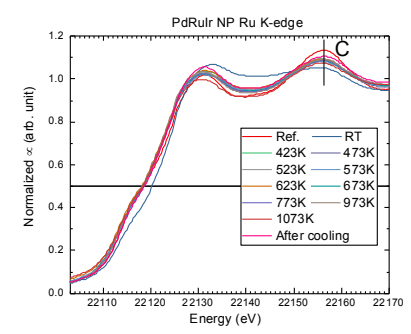

(c)

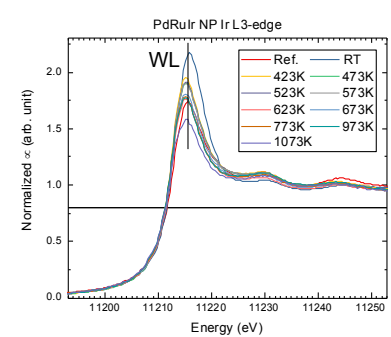

(f)

Figure S5. XANES spectra of samples measured at Ru K-edge (a,b,c), Pd K-edge (d,e) and Ir $\mathrm{L}_{3}$-edge (f) for Ru NPs (a), PdRu NPs (b,d) and PdRuIr NPs (c,e,f). 
Synthesis of nanoparticles:

Mono and bimetallic NPs were prepared using the chemical reduction method with $\mathrm{K}_{2} \mathrm{PdCl}_{4}$ and $\mathrm{RuCl}_{3} \cdot \mathrm{nH}_{2} \mathrm{O}$ as the metal precursors, as described earlier. [1-2] The ternary PdRuIr NPs are prepared by dissolving Poly(N-vinyl-2-pyrrolidone) (PVP) in triethylene glycol (TEG). The solution was then heated in the air under magnetic stirring at $503 \mathrm{~K} . \mathrm{K}_{2} \mathrm{PdCl}_{4}$, $\mathrm{RuCl}_{3} \cdot \mathrm{nH}_{2} \mathrm{O}$, and $\mathrm{IrCl}_{4} \cdot \mathrm{nH}_{2} \mathrm{O}$ dissolved in deionized water were then added to the TEG solution kept at $503 \mathrm{~K}$. After cooling, the NPs are separated using centrifugation and using acetone and diethyl ether. [3] The PVP was added as the stabilizing agent.

Reference:

1. C. Song et al., Correlation between the electronic/local structure and CO-oxidation activity of $\mathrm{Pd}_{\mathrm{x}} \mathrm{Ru}_{1-\mathrm{x}}$ alloy nanoparticles. Nanoscale Advances (2018) DOI: 10.1039/c8na00305j.

2. K. Kusada et al., Solid Solution Alloy Nanoparticles of Immiscible Pd and Ru Elements Neighboring on Rh: Changeover of the Thermodynamic Behavior for Hydrogen Storage and Enhanced CO-Oxidizing Ability. J. Am. Chem. Soc. (2014) 136, 1864-1871.

3. K. Kusada et al., Highly Stable and Active Solid-Solution-Alloy Three-Way Catalyst by Utilizing Configurational-Entropy Effect. Adv. Mater. (2021) 33, 2005206. 